\title{
Effect of pulsed electromagnetic field treatment on programmed resolution of inflammation pathway markers in human cells in culture
}

This article was published in the following Dove Press journal:

Journal of Inflammation Research

23 February 2015

Number of times this article has been viewed

\section{Nicole J Kubat' \\ John Moffett ${ }^{2}$ \\ Linley M Fray ${ }^{2}$}

'Nicole Kubat Consulting, Pasadena, CA, USA; '2Life Science Department, Regenesis Biomedical, Inc., Scottsdale, AZ, USA
Correspondence: John Moffett Regenesis Biomedical, Inc., 530 I North Pima Road, Scottsdale, AZ 85250-3773 USA

Tel + I 4809704970

Fax + I 8668578792

Email moffett@regenesisbio.com
Abstract: Inflammation is a complex process involving distinct but overlapping biochemical and molecular events that are highly regulated. Pulsed electromagnetic field (PEMF) therapy is increasingly used to treat pain and edema associated with inflammation following surgery involving soft tissue. However, the molecular and cellular effects of PEMF therapy on pathways involved in the resolution of inflammation are poorly understood. Using cell culture lines relevant to trauma-induced inflammation of the skin (human dermal fibroblasts, human epidermal keratinocytes, and human mononuclear cells), we investigated the effect of PEMF on gene expression involved in the acute and resolution phases of inflammation. We found that PEMF treatment was followed by changes in the relative amount of messenger (m)RNAs encoding enzymes involved in heme catabolism and removal of reactive oxygen species, including an increase in heme oxygenase 1 and superoxide dismutase 3 mRNAs, in all cell types examined 2 hours after PEMF treatment. A relative increase in mRNAs encoding enzymes involved in lipid mediator biosynthesis was also observed, including an increase in arachidonate 12- and 15-lipoxygenase mRNAs in dermal fibroblasts and epidermal keratinocytes, respectively. The relative amount of both of these lipoxygenase mRNAs was elevated in mononuclear cells following PEMF treatment relative to nontreated cells. PEMF treatment was also followed by changes in the mRNA levels of several cytokines. A decrease in the relative amount of interleukin 1 beta mRNA was observed in mononuclear cells, similar to that previously reported for epidermal keratinocytes and dermal fibroblasts. Based on our results, we propose a model in which PEMF therapy may promote chronic inflammation resolution by mediating gene expression changes important for inhibiting and resolving inflammation.

Keywords: acute inflammation, resolution phase, heme oxygenase, reactive oxygen species, eicosanoids, resolvins, cytokines, lipid mediators

\section{Introduction}

Inflammation, an integral physiological response to trauma, is a complex, highly regulated process. ${ }^{1-3}$ Its temporal nature - an acute phase (proinflammatory), followed by a dampening of the acute response (anti-inflammatory), and finally a return to homeostasis (resolution) - involves timely and spatially regulated action of the molecules and cell types involved. ${ }^{4}$

The acute inflammatory response is typified by a localized cocktail of inflammatory factors, including cytokines, growth factors, eicosanoids, neuroactive factors, heme, proteases, and reactive oxygen species. ${ }^{5-9}$ Chemotactic influx of neutrophils and leukocytes is promoted by a local increase in vascular permeability, for example, in response to prostaglandins $\mathrm{E}_{2}$ and $\mathrm{I}_{2}$, and chemoattracting factors at the site of injury, such as 
heme, leukotriene B4, and chemokines. ${ }^{10}$ Several acute-phase inflammatory compounds are responsible for evoking pain and edema, leading to erythema and tissue swelling. ${ }^{11}$

Dampening of the inflammatory response relies on the anti-inflammatory action of various factors. Cytokines with anti-inflammatory effects, such as interleukin 10, are well-known examples. ${ }^{12}$ Regulation of free heme through sequestration or catabolism is essential for the resolution of inflammation, which is controlled by haptoglobin, or intracellularly catabolized by heme oxygenase (Figure 1). ${ }^{13-15}$ Heme oxygenase is thought to act as a molecular switch that leads to the resolution of inflammation by the degradation of heme to the anti-inflammatory molecules biliverdin and carbon monoxide. ${ }^{14}$ Limiting the inflammatory response further depends on factors with dual pro- and anti-inflammatory effects. For example, prostaglandins play a major role in inflammation and are key agents involved in eliciting pain during peripheral injury by binding to prostaglandin receptors expressed at the cell membrane of nociceptive sensory neurons. ${ }^{16-18}$ On the other hand, their production, namely that of prostaglandins $\mathrm{D}_{2}$ and $\mathrm{E}_{2}$, is important for initiating "lipid mediator class switching" from the production of proinflammatory mediators to proresolving ones (Figure 1). ${ }^{10,16,19-22}$

Active resolution of the inflammatory phase is promoted by proresolving lipid mediators derived from omega-3 and - 6 polyunsaturated fatty acids, including arachadonic acidderived lipoxin A4, docosahexaenoic acid-derived resolvins, protectins, and maresins, and eicosapentaenoic acid-derived resolvins (Figure 1). Proresolving lipid mediators actively mediate a return to homeostasis. Lipoxin A4, for example,

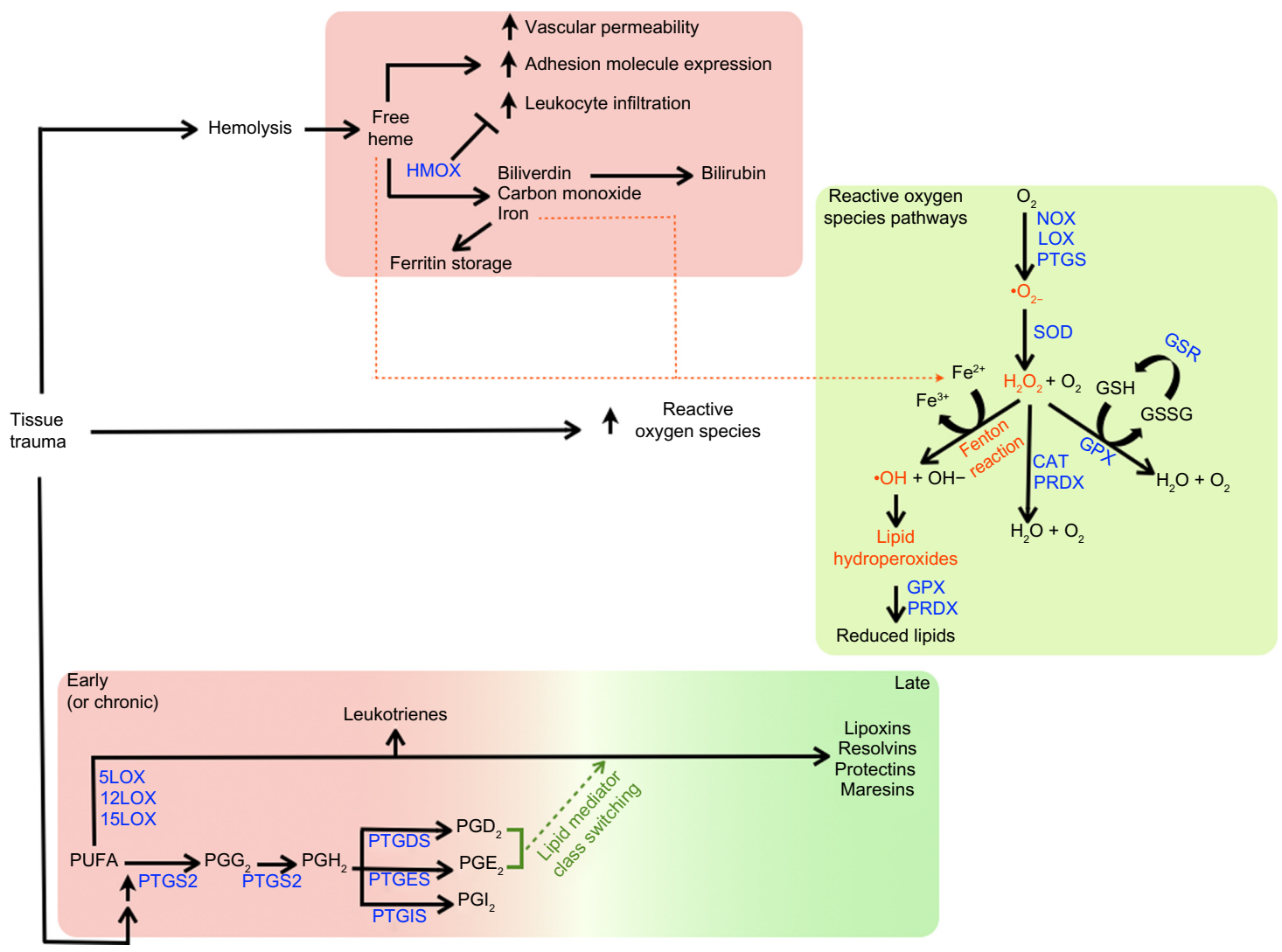

Figure I Consequences of tissue trauma on heme catabolism, reactive oxygen species, and polyunsaturated fatty acid metabolism.

Notes: Top panel: following tissue trauma, release of free heme can serve as a signal for proinflammatory events. ${ }^{71}$ The oxidative degradation of heme by heme oxygenase removes free heme, and generates catabolites with antioxidant and anti-inflammatory effects. ${ }^{70-75}$ Iron released from heme by heme oxygenase catabolism can be stored by ferritin, preventing its participation in redox reactions. ${ }^{70}$ Middle panel: Acute inflammation is associated with an increase in reactive oxygen species, which are regulated by cellular enzymes involved in redox reactions..$^{55,76,77}$ Reactive oxygen species can have proinflammatory effects, and chronic inflammatory diseases are often associated with oxidative stress. ${ }^{55,76,77,79}$ In some cases, reactive oxygen species can have anti-inflammatory effects. ${ }^{62,78}$ Lower panel: in response to proinflammatory stimuli, phospholipase A2 mediates release of arachidonic acid from the cell membrane. ${ }^{80,81}$ An increase in inducible prostaglandin-endoperoxide synthase 2 also occurs. ${ }^{80,82-84}$ During the early inflammatory response, arachidonic acid is metabolized by prostaglandin synthases and lipoxygenases to generate eicosanoids and leukotrienes, ${ }^{21,24,85-87}$ which is followed, at later times, by "lipid mediator class switching." The latter is thought to serve as a switch from the production of proinflammatory lipid mediators to those involved in programmed resolution. ${ }^{10,16,19-22}$

Abbreviations: $G S H$, reduced glutathione; GSSG, glutathione disulfide; $P \mathrm{PD}_{2}$, prostaglandin $\mathrm{D}_{2}$; $\mathrm{PGE}_{2}$, prostaglandin $\mathrm{E}_{2}$; $P \mathrm{GG}_{2}$, prostaglandin $\mathrm{G}_{2}$; $P \mathrm{PH}_{2}$, prostaglandin $\mathrm{H}_{2}$; $\mathrm{PGI}_{2}$, prostaglandin $\mathrm{I}_{2}$; PUFA, polyunsaturated fatty acid. 
Table I List of messenger RNAs evaluated in the study

\begin{tabular}{ll}
\hline Name & Abbreviation \\
\hline Heme oxygenase (decycling) I (alias: HO-I) & HMOXI \\
Heme oxygenase (decycling) 2 (alias: HO-2) & HMOX2 \\
NADPH oxidase I & NOXI \\
Catalase & CAT \\
Glutathione peroxidase 3 & GPX3 \\
Glutathione reductase & GSR \\
Peroxiredoxin 6 & PRDX6 \\
Superoxide dismutase 3 & SOD3 \\
Prostaglandin-endoperoxide synthase I (prostaglandin & PTGSI \\
G/H synthase and cyclooxygenase) (alias: COX-I) & \\
Prostaglandin-endoperoxide synthase 2 (prostaglandin & PTGS2 \\
G/H synthase and cyclooxygenase) (alias: COX-2) & \\
Prostaglandin D2 synthase & PTGDS \\
Prostaglandin E synthase & PTGES \\
Prostaglandin I2 (prostacyclin) synthase & PTGIS \\
Arachidonate 5-lipoxygenase & ALOX5 \\
Arachidonate I2-lipoxygenase & ALOXI2 \\
Arachidonate I5-lipoxygenase & ALOXI5 \\
Interleukin I, alpha & ILIA \\
Interleukin I, beta & ILIB \\
Interleukin 2 & IL2 \\
Interleukin 5 & IL5 \\
Interleukin 6 & IL6 \\
Interleukin I0 & ILI0 \\
Interleukin I2B & ILI2B \\
Interleukin 20 & IL20 \\
Interleukin 2I & IL2I \\
\hline Notes: Nam and abbeval & \\
\hline
\end{tabular}

Notes: Names and abbreviations correspond to HGNC-approved names and symbols. For some names, common aliases are also provided in parentheses. Abbreviation: HGNC, HUGO Gene Nomenclature Committee.

serves as a "stop" signal for leukocyte chemotaxis, vascular permeability, vasodilation, pain, and hyperalgesia, while the resolvins have been found to prevent polymorphonuclear cell infiltration. ${ }^{19,23-25}$

Inflammatory pain is a notable clinical issue. During cutaneous injury, cells from the immune system and peripheral organs express factors that both promote pain as a selfprotective measure, as well elicit an analgesic response. As alluded to previously, in several cases, this dichotomy of cellular responses involves pleiotrophic factors that can act as proinflammatory, proalgesic agents, while at later times, may have anti-inflammatory or analgesic effects, depending on the molecular and cellular environment. ${ }^{26,27}$ Chronic pain associated with deregulation of the acute inflammatory response and the development of chronic debilitating inflammation is a clinical condition that is challenging to treat. Fully understanding the molecular events that lead to chronic inflammation as opposed to resolution is essential to the development of better treatment strategies for chronic inflammation and inflammatory pain..$^{28,29}$
New anti-inflammatory treatments are actively being developed to treat inflammatory pain. Because of the complexity of inflammation, many pharmaceuticals treat one physiological symptom or molecular pathway of the process. ${ }^{30}$ Opioids are commonly used for the treatment of severe pain. ${ }^{31}$ Although they are extremely effective for the treatment of acute pain, when used for extended periods in patients with chronic inflammation, severe side effects become a major drawback. ${ }^{32}$ The nonsteroidal antiinflammatory drugs function by blocking prostaglandin synthesis, via inhibition of prostaglandin-endoperoxide synthase 1 and 2 (ie, cyclooxygenase-1 and -2), and are effective pharmaceuticals for short-term acute inflammation relief..$^{33}$ A relatively new series of pharmaceuticals include specialized therapies that target specific mediators of the inflammation response. ${ }^{34-36}$ These therapeutics usually target proinflammatory cytokines or specific receptors on immune cells to inhibit inflammation. Because of the side effects of these pharmaceuticals and their inability to promote the resolution of inflammation, new modalities are needed.

One emerging therapy for the treatment of both acute and chronic inflammation is the application of pulsed electromagnetic fields (PEMF). ${ }^{37-42}$ PEMF therapy involves application of pulsed electromagnetic energy to inflamed superficial soft tissue; for PEMF in the radio frequency range, the carrier frequency is typically $27.12 \mathrm{MHz} .{ }^{43}$ It is delivered via a noninvasive medical device treatment applicator placed adjacent to the tissue target and, unlike some other radio frequency-based therapies, is not intended to generate deep heat. The treatment has been associated with postoperative pain relief in randomized, double-blind, sham-controlled pilot trials following breast surgery ${ }^{39-41}$ and recently, in a single-arm, open-label pilot study of failed back surgery syndrome pain. ${ }^{38} \mathrm{~A}$ meta-analysis assessing the effectiveness of PEMF therapy in clinical studies found statistical support for efficacy of the therapy in treating postoperative pain and edema. ${ }^{44}$ Technical parameters of the technology, as well as a summary of clinical studies performed using the therapy, have been recently reviewed. ${ }^{45}$ Though also referred to as "pulsed radio frequency energy", the term PEMF therapy will be used to avoid confusion with "radio frequency ablation," which unlike the therapy described here, involves localized thermal ablation of tissue via a radiofrequency electrode inserted into tissue. ${ }^{46}$

The primary mechanism of action of PEMF-mediated analgesia is hypothesized to involve promoting the reduction and, possibly, resolution of inflammation. ${ }^{47-51}$ Results from 
in vitro and in vivo studies ${ }^{52,53}$ indicate that PEMF therapy may regulate gene expression by influencing the activation of ion channels. Recent results in rodents have indicated that both $\mathrm{Na}^{+}$and $\mathrm{Ca}^{2+}$ voltage-gated ion channels are activated by extremely low frequency electromagnetic fields. ${ }^{52}$ Activation of these channels leads to activation of second messenger pathways and downstream regulation of gene expression. ${ }^{53}$ Previous studies in vitro have also found that PEMF treatment of cells in culture is followed by changes in the gene expression profiles of a number of factors associated with inflammation and analgesia, ${ }^{47,48}$ including an increase in endogenously expressed opioid precursors, both at the messenger (m)RNA and peptide levels. ${ }^{48}$ Additionally, results of a clinical pilot study found significantly lower levels of interleukin 1 beta peptide in postoperative wound exudates from PEMF-treated tissue relative to sham-treated tissue, which corresponded with lower pain scores in the PEMF treatment group. ${ }^{41}$ Together, these findings suggest that PEMF-mediated changes in gene expression underlie the anti-inflammatory and analgesic effects associated with PEMF therapy.

In light of these findings, it was of interest to further investigate the effect of PEMF treatment on gene expression relevant to inflammation and in particular, factors involved in repressing or resolving inflammation caused by trauma, notably trauma to the skin. In this study, we evaluated the effect of PEMF treatment on human dermal fibroblasts (HDF), epidermal keratinocytes (HEK), and mononuclear cells (HMNC) in culture by assessing changes in mRNA levels of enzymes involved in heme catabolism, lipid mediator biosynthesis, and reduction of reactive oxygen species (Figure 1), as well as cytokines involved in the inflammation process. Our results support a model in which PEMF treatment promotes the resolution of inflammation by stimulating the expression of cytokines and metabolic pathways involved in dampening and resolving the inflammatory response.

\section{Material and methods Materials}

Culture media were purchased from Cell Applications, Inc. (San Diego, CA, USA) and from Mediatech, Inc. (Herndon, VA, USA) for general culture of HDF, HEK, and HMNC cells. Fetal calf serum, penicillin/streptomycin, trypsin, $1 \mathrm{X}$ PBS, sodium pyruvate, and nonessential amino acids were purchased from Hyclone (Logan, UT, USA) Reagents for quantitative reverse-transcription (qRT) and polymerase chain reaction (PCR) were from Quanta Biosciences (Gaithersburg, MD, USA). Oligonucleotide primers for PCR were purchased from/synthesized by Real Time Primers, LLC
(Elkins Park, PA, USA). General chemicals were purchased from Sigma-Aldrich Corp (St Louis, MO, USA).

\section{Cell culture}

Adult HDF, HEK, and HMNC were purchased from Cell Applications, Inc. (San Diego, CA, USA). Routine culture was performed as described in Moffett et al. ${ }^{48}$ For HDF experiments, cells were cultured in $10 \mathrm{~cm}$ plates at a density of $1.14 \times 10^{4}$ cells $/ \mathrm{cm}^{2}$ in minimum essential media supplemented with $1 \mathrm{mM}$ sodium pyruvate, $1 \mathrm{mM}$ nonessential amino acids, 100 units penicillin, $100 \mu \mathrm{g}$ streptomycin, and 5\% fetal calf serum. Cells were used for experimentation after 16 hours in a humidified atmosphere at $37^{\circ} \mathrm{C}$ with $5 \% \mathrm{CO}_{2}$. Cells were used for experimentation from passage 5 to 10 . Routine culture was performed, as recommended by the manufacturer, in a $5 \% \mathrm{CO}_{2}$ humidified atmosphere at $37^{\circ} \mathrm{C}$. For experiments, keratinocytes were cultured in $10 \mathrm{~cm}$ plates at a density of $0.75 \times 10^{4}$ cells $/ \mathrm{cm}^{2}$ in keratinocyte growth media, supplied by the manufacturer. HEK were used for experimentation after 16 hours in a humidified atmosphere at $37^{\circ} \mathrm{C}$ with $5 \% \mathrm{CO}_{2}$. HEK were used for experimentation from passage 5 to 7 . HMNC cells were cultured, as recommended by the manufacturer, in a $5 \% \mathrm{CO}_{2}$ humidified atmosphere at $37^{\circ} \mathrm{C}$.

\section{PEMF conditions and treatment}

Treatments were performed by exposing cells to the PEMF field from a Provant ${ }^{\circledR}$ device from Regenesis Biomedical, Inc. (Scottsdale, AZ, USA). This device emits a 27.12 MHz radio frequency signal transmitted from a flat spiral antenna with a radius of $7.5 \mathrm{~cm}$, made up of six turns at a width of $0.70 \mathrm{~cm}$, spaced with a separation of $0.3 \mathrm{~cm}$ (Figure 2). The signal is delivered in $42 \mu \mathrm{sec}$ pulses with a period of $1 \mathrm{KHz}$. This corresponds to energy parameters of $591 \mathrm{~V} / \mathrm{m}$ and $6.7 \mathrm{~A} / \mathrm{m}$ in electrical and magnetic fields, respectively, when measured $5 \mathrm{~cm}$ from the surface of the radiating antenna. Cells in cell culture medium were placed at a distance of $5 \mathrm{~cm}$ from the source during treatment. Treatment was performed at room temperature for 30 minutes, and then cells were returned to the incubator. Cells were harvested for total RNA at the times indicated in the figures. Treatment under these conditions will be referred to as "PEMF treatment".

\section{RNA isolation and real-time qRT-PCR}

Total RNA was isolated using an RNeasy Mini kit (Qiagen, Hilden, Germany). Total RNA (0.05 $\mu \mathrm{g})$ was reverse-transcribed using a high capacity RNA to complementary (c)DNA master mix (Quanta Biosciences) following the manufacturer's instructions. PCR was performed 


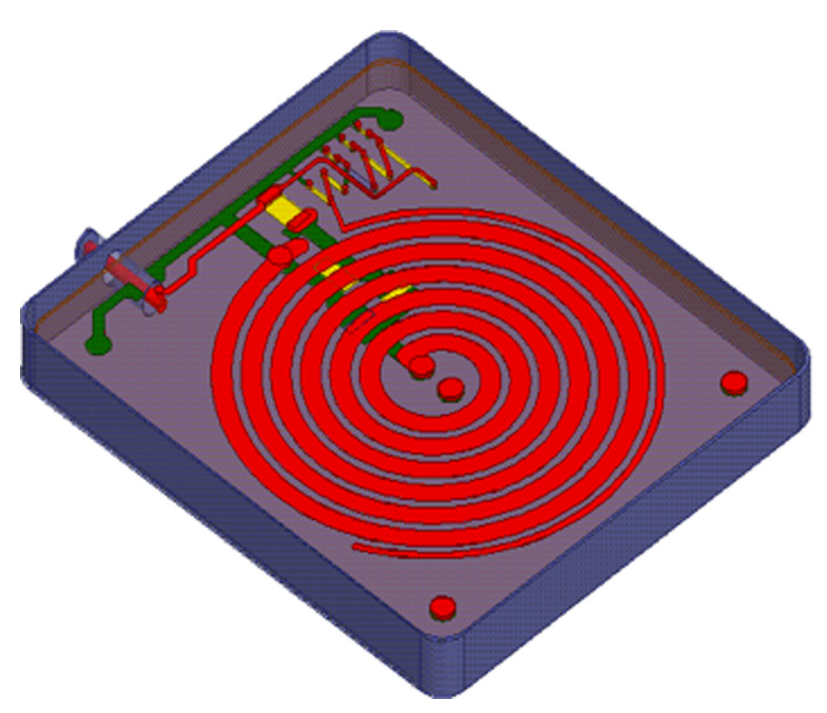

Figure 2 An illustration of the device's spiral antenna.

using the following reaction times and temperatures: denaturation for 15 seconds at $94^{\circ} \mathrm{C}$, annealing for 30 seconds at $55^{\circ} \mathrm{C}$, and elongation for 30 seconds at $72^{\circ} \mathrm{C}$; in $20 \mu \mathrm{L}$ reaction volumes and run out to 45 cycles using a Rotogene device (Qiagen). mRNAs evaluated in the study are listed in Table 1. Primer oligonucleotides sequences for prostaglandin-endoperoxide synthase 1 , prostaglandin-endoperoxide synthase 2, prostaglandin $\mathrm{E}$ synthase, interleukin 1 beta, interleukin 6 , and interleukin 10 were reported in Moffett et al. ${ }^{48}$ All other primer sequences are available from the manufacturer (Real Time Primers, LLC). Quantitation and analysis of relative gene expression was performed by the $2^{-\Delta \Delta \mathrm{CT}}$ method as described by Livak and Schmittgen. ${ }^{54}$ GAPDH was used to normalize product expression between different RNA samples and treatments. RNA was isolated from at least four independent experiments, and PCR was performed in triplicate. Products were electrophoresed on $2 \%$ agarose gels and stained with ethidium bromide to determine the fidelity of the PCR reaction. The relationship between the concentration of input RNA and the amount of PCR product was linear for each PCR product.

\section{Statistical analysis}

qRT-PCR data were analyzed using one-way analysis of variance (ANOVA), using SigmaPlot version 11.0 (Systat Software, Inc., San Jose, CA, USA). If the equal variance test failed for one-way ANOVA, the Dunn's method for multiple comparisons was used (SigmaPlot version 11.0). For all experiments reported, a $P$-value of less than 0.05 was considered significant.

\section{Results \\ PEMF treatment of HDF, HEK, and $\mathrm{HMNC}$ is followed by an increase in heme oxygenase mRNA}

One of the first proinflammatory compounds at the site of trauma is heme, which is released from heme carrier proteins, such as hemoglobin and myoglobin, during tissue damage and cellular lysis (Figure 1). The inducible isoform of the enzyme responsible for the degradation of heme, heme oxygenase 1 , is regulated by a number of compounds and environmental stressors, including cytokines, heavy metals, hypoxia, and heme itself. To evaluate whether PEMF might also affect heme oxygenase expression, we treated cultured HDF, HEK, and HMNC with PEMF for 30 minutes and measured changes in heme oxygenase mRNA levels following treatment. A rapid increase in heme oxygenase $1 \mathrm{mRNA}$ was observed in all cell types following PEMF treatment relative to untreated cells (Figure 3A and Table 2). Timing of maximum induction was 1.5 hours after the end of treatment for all three cell types, with a fold increase relative to untreated cells ranging from 2.5- to 2.9-fold, depending on the cell type. In contrast, the level of heme oxygenase 2 mRNA remained unchanged in all three cell types following treatment (Figure 3B).

\section{The effect of PEMF on pro- and antioxidant enzyme mRNAs in HDF, HEK, and HMNC}

Proinflammatory reactive oxygen species can be generated from a number of sources during the inflammation process. Oxidation of divalent iron, a product of heme catabolism, can result in the generation of reactive oxygen species via the Fenton reaction (Figure 1). Proinflammatory stimuli, such as tumor necrosis factor alpha, can lead to increased reactive oxygen species-generation by mitochondrial enzyme complexes, as well as by membrane-associated NADPH oxidase, in both phagocytic and nonphagocytic cells. ${ }^{55}$ Reactive oxygen species can also be generated during leukotriene synthesis. ${ }^{56}$ Given the importance of antioxidant enzymes in the removal of reactive oxygen species, we examined what effect, if any, PEMF had on expression levels of antioxidant enzymes. PEMF treatment was followed by increased levels of superoxide dismutase 3 and peroxiredoxin 6 mRNAs in HDF, HEK, and HMNC cells relative to untreated cells (Figure 4 and Table 2). Glutathione reductase mRNA levels were greater in HDF and HMNC following PEMF treatment, while the relative amount of catalase mRNA was greater 
A

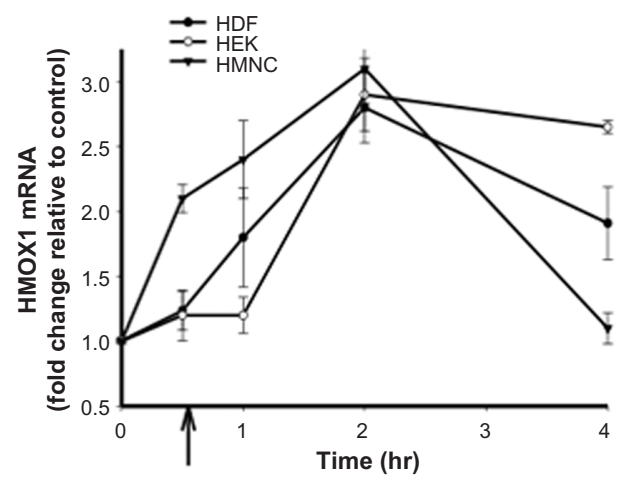

B

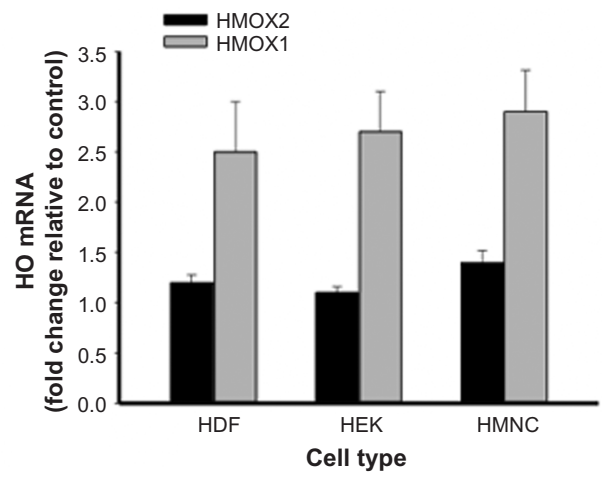

Figure 3 The effect of PEMF treatment on heme oxygenase mRNA levels in HEK, HDF, and HMNC in culture.

Notes: Cells were treated with PEMF for 30 minutes, after which total RNA was isolated and mRNAs quantitated at several time points by qRT-PCR. Cells were harvested at the time points indicated (A) or 2 hours postinitiation of PEMF treatment (B). In (A), the $x$ axis indicates the amount of time passed after the initiation of PEMF treatment, with the end of the 30-minute treatment period indicated by an arrow. In (B), the results of three independent experiments with PEMF are shown. All data is expressed relative to untreated control cell cultures.

Abbreviations: HDF, human dermal fibroblasts; HEK, human epidermal keratinocytes; HMNC, human mononuclear cells; mRNA, messenger RNA; PEMF, pulsed electromagnetic field; qRT-PCR, quantitative reverse-transcription polymerase chain reaction.

after PEMF treatment in HDF and HEK cells. In contrast, no change in glutathione peroxidase $3 \mathrm{mRNA}$, in any of the three cell types, was observed following PEMF treatment compared with untreated cells (Figure 4). Interestingly, in addition to an increase in RNA transcripts encoding antioxidant enzymes, PEMF treatment was also followed by a relative increase in NADPH oxidase mRNA, a prooxidant enzyme, in HDF and HMNC (Figure 4 and Table 2).

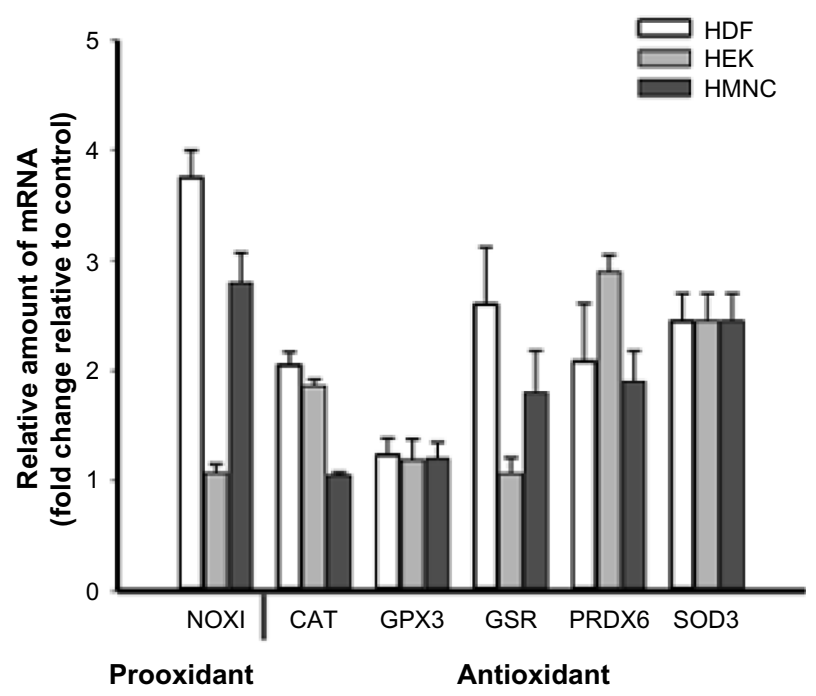

Figure 4 Changes in cellular redox enzyme mRNA levels following PEMF treatment of HDF, HEK, and HMNC.

Notes: Cells were treated with PEMF for 30 minutes, harvested 2 hours after treatment, and relative mRNA levels were determined by qRT-PCR. All data are expressed relative to untreated control cell cultures. $\mathrm{n}=6$.

Abbreviations: HDF, human dermal fibroblasts; HEK, human epidermal keratinocytes; HMNC, human mononuclear cells; mRNA, messenger RNA; PEMF, pulsed electromagnetic field; qRT-PCR, quantitative reverse-transcription polymerase chain reaction.

\section{The effect of PEMF treatment on mRNA levels of enzymes involved in lipid mediator biosynthesis}

A number of metabolites of omega-3 and -6 polyunsaturated fatty acids, such as prostaglandins, leukotrienes, lipoxins, resolvins, protectins, and maresins, function as bioactive lipid mediators, with important roles during both the acute and resolution phases of inflammation (Figure 1). To better understand the possible effects of PEMF treatment on lipid mediator biosynthesis, we measured relative mRNA levels of enzymes involved in lipid mediator synthesis in cells following PEMF treatment compared with untreated cells. A rapid rise (15 minutes following PEMF treatment), followed by a decrease, in both prostaglandin-endoperoxide synthases 1 and 2 mRNA levels was observed in HMNC following PEMF treatment relative to levels observed in untreated control cells (data not shown). Similar to a previous study, ${ }^{48}$ an increase in prostaglandin-endoperoxide synthase 2 expression was found in HDF following PEMF treatment, with relatively little change in the level of either transcript observed in HEK after PEMF treatment (Figure 5A and Table 2). The effect of PEMF treatment on expression of downstream prostaglandin synthases was also assessed. The relative amount of prostaglandin E synthase mRNA appeared to be most affected by PEMF treatment in HDF and HMNC (Figure 5A and Table 2). A PEMF-associated increase in prostaglandin E synthase mRNA in HDF, but not HEK, is in line with previously reported findings. ${ }^{48}$ While mRNA levels of several downstream prostaglandin synthases were upregulated in $\mathrm{HDF}$ and HMNC following PEMF treatment, only prostaglandin I2 
Table 2 Statistically meaningful changes in mean mRNA levels detected within 4 hours after PEMF treatment relative to untreated control cells

\begin{tabular}{|c|c|c|c|}
\hline & HDF & HEK & HMNC \\
\hline Heme oxygenases & 个HMOXI* & 个HMOXI* & 个HMOXI** \\
\hline \multirow[t]{5}{*}{ Cellular redox enzymes } & 个NOXI* & & 个NOXI \\
\hline & 个CAT & 个СAT & \\
\hline & 个GSR & & 个GSR \\
\hline & 个PRDX6 & 个PRDX6 & 个PRDX6 \\
\hline & 个SOD3 & 个SOD3 & 个SOD3 \\
\hline \multicolumn{4}{|c|}{ Lipid metabolism enzymes } \\
\hline Prostaglandin & 个PTGS2 & & \\
\hline \multirow[t]{3}{*}{ synthesis enzymes } & 个PTGDS & & 个PTGDS \\
\hline & 个PTGES* & & 个PTGES \\
\hline & 个PTGIS & 个PTGIS & 个PTGIS \\
\hline \multirow[t]{2}{*}{ Lipoxygenases } & 个ALOXI2 & & 个ALOXI2 \\
\hline & & 个ALOXI5 & 个ALOXI5 \\
\hline \multirow[t]{6}{*}{ Interleukins } & $\downarrow$ \ILIB* & $\downarrow$ ฟILIB & $\begin{array}{l}\downarrow \text { ILIB } \\
\downarrow_{\text {IL5 }}\end{array}$ \\
\hline & 个IL6 & 个IL6 & \\
\hline & 个ILI0* & 个ILIO* & \\
\hline & 个ILI2B & & TILI2B \\
\hline & & & 个IL20 \\
\hline & & & 个IL2I \\
\hline
\end{tabular}

Notes: $P<0.05$ or better were met in all cases; $P<0.01$ and greater denoted by asterisks $(* P<0.0 \mathrm{I}$ and $* * P<0.00 \mathrm{I})$. An increase or decrease in relative mRNA levels is indicated by upward- and downward-facing arrows, respectively.

Abbreviations: HDF, human dermal fibroblasts; HEK, human epidermal keratinocytes; HMNC, human mononuclear cells; mRNA, messenger RNA; PEMF, pulsed electromagnetic field.

(prostacyclin) synthase was upregulated in HEK at the time point examined (Figure 5A and Table 2).

Next, we examined the effect of PEMF treatment on lipoxygenase mRNA levels in HDF, HEK, and HMNC. PEMF treatment of HDF, HEK, or HMNC cells had no effect on arachidonate 5-lipoxygenase mRNA levels (Figure 5B). In contrast, PEMF treatment of HDF and HMNC cells was associated with an increase in arachidonate 12-lipoxygenase mRNA relative to untreated cells (three- and 2.5-fold, respectively) (Figure 5B and Table 2). A threefold increase in arachidonate 15-lipoxygenase mRNA was also observed in HMNC and HEK after PEMF treatment relative to untreated cells (Figure 5B and Table 2).

\section{The effect of PEMF treatment on mRNAs of cytokines with pro- and anti-inflammatory effects}

Cytokine-mediated effects on inflammation are often microenvironment-dependent and can vary depending on other cytokines and regulatory molecules present. We have previously shown that PEMF treatment of HEK and HDF cells is associated with changes in mRNA levels of cytokines involved in regulating inflammatory pain and the inflammation phase of wound healing. ${ }^{48,57}$ To further investigate PEMF-associated changes in cytokine expression profiles, we measured relative mRNA levels of several cytokines in HDF, HEK, and HMNC following PEMF treatment. Interleukin 1 beta mRNA levels were lower in all three cell types following PEMF treatment (Figure 6 and Table 2), in agreement with previous findings in HEK and HDF cells. ${ }^{48}$ The expression profiles of several other cytokine mRNAs changed following PEMF treatment as well, which varied by cell type. Interleukin 1 alpha mRNA levels were lower in HDF and HMNC after PEMF treatment relative to untreated control cells, though remained unchanged in PEMF-treated HEK at the time point examined (Figure 6). While interleukin 5 mRNA levels were greater in PEMF-treated HEK relative to untreated HEK, PEMF-treated HMNC had lower levels of interleukin 5 mRNA at the same time point relative to untreated HMNC (Figure 6 and Table 2). PEMF treatment was followed by an increase in mRNA levels of interleukin 12B in HDF and HMNC, while mRNA levels of interleukins 20 and 21 were upregulated only in HEK and HMNC relative to untreated control cells (Figure 6 and Table 2).

\section{Discussion}

Inflammation is an essential response by tissues to environmental challenges, both pathogens and injury. ${ }^{1,4,11,27}$ This process is divided into two broad phases, acute inflammation and resolution, which are regulated by tightly controlled programs of gene expression. ${ }^{29,58,59}$ Although the inflammatory response is protective in nature, dysregulation of the acute or resolution phases can lead to chronic inflammation and disease states.

Postoperative inflammation can involve the skin and underlying soft tissue. PEMF therapy has been associated with improvement in postoperative pain in clinical trials, ${ }^{38-41}$ which studies suggest may be related to molecular changes impacting inflammation and inflammatory pain. ${ }^{37,41-43,48,57}$ To further investigate this possibility in the current study, we used cell culture lines derived from human skin (HDF and HEK), as well as HMNCs, to determine the effect of PEMF treatment on mRNA levels of gene products relevant to trauma-induced inflammation of the skin, which can develop into a chronic problem in those with metabolic disorders. PEMF treatment of HDF, HEK, and HMNC was followed by changes in relative mRNA levels of several factors involved in suppressing the acute inflammatory response and mediating active resolution of inflammation. Table 2 provides a summary of statistically significant 
A

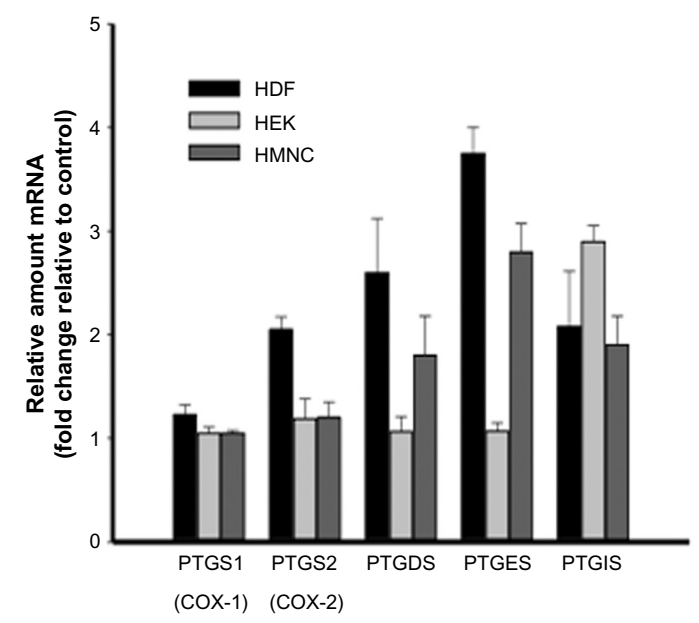

B

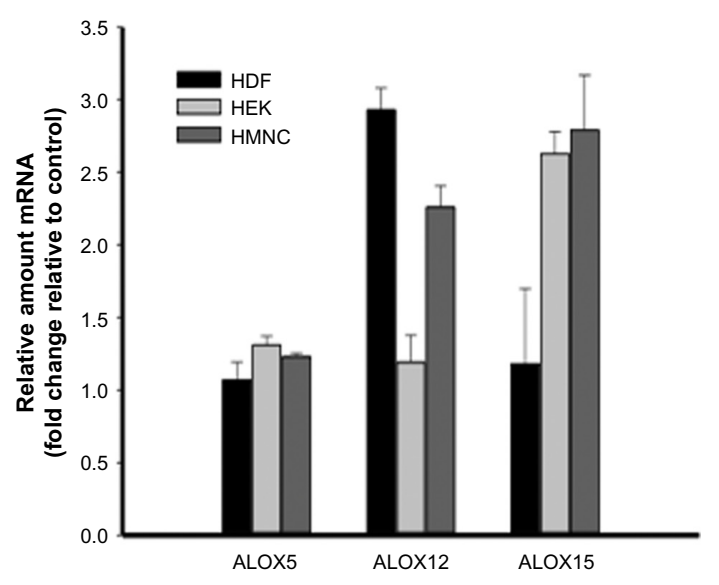

Figure 5 The effect of PEMF on RNA message levels of enzymes involved in lipid mediator synthesis.

Notes: HEK, HDF, and HMNC were treated with PEMF for 30 minutes, after which relative amounts of mRNAs encoding (A) prostaglandin synthases and (B) lipoxygenases were determined using QRT-PCR. Cells were harvested 4 hours $(\mathbf{A})$ or 2 hours $(\mathbf{B})$ after initiation of PEMF treatment. $n=6$.

Abbreviations: HDF, human dermal fibroblasts; HEK, human epidermal keratinocytes; HMNC, human mononuclear cells; mRNA, messenger RNA; PEMF, pulsed electromagnetic field; qRT-PCR, quantitative reverse-transcription polymerase chain reaction.

changes observed in the current study. Heme oxygenase 1 mRNA levels were increased in all cell types following PEMF treatment. Following trauma, free heme can serve as a proinflammatory signal, and thus, its removal is important for abating the inflammatory response. ${ }^{14,15,60,61}$ We also found that PEMF treatment was associated with increased expression of several enzymes that neutralize

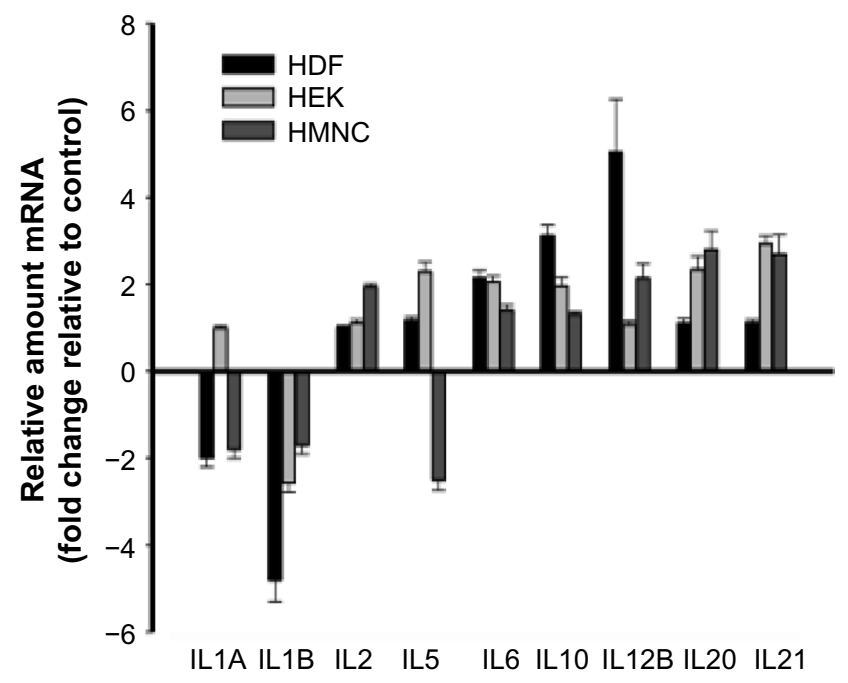

Figure 6 The effect of PEMF treatment on cytokine mRNA levels.

Notes: HEK, HDF, or HMNC were treated with PEMF for 30 minutes. Total RNA was isolated 2 hours after PEMF treatment, and relative amounts of cytokine mRNA were determined using qRT-PCR. $n=6-9$. All data is expressed relative to untreated control cell cultures.

Abbreviations: HDF, human dermal fibroblasts; HEK, human epidermal keratinocytes; HMNC, human mononuclear cells; mRNA, messenger RNA; PEMF, pulsed electromagnetic field; qRT-PCR, quantitative reverse-transcription polymerase chain reaction. oxygen radicals. Interestingly, expression of the prooxidant enzyme, NADPH oxidase 1, was also upregulated in HDF and HMNC after PEMF treatment. Though reactive oxygen species generated by mitochondria have been implicated in promoting inflammation in sterile inflammation, reactive oxygen species generated by cytoplasmic NADPH oxidases may be anti-inflammatory during sterile inflammatory responses. ${ }^{62}$

During acute inflammation, resident and recruited cells alike produce proinflammatory mediators, including eicosanoids and cytokines. ${ }^{63,64}$ While prostaglandin synthases and lipoxygenases are involved in generating eicosanoids and leukotrienes with proinflammatory effects, they are also necessary for production of lipid mediators involved in programmed resolution, ${ }^{65,66}$ namely those that promote lipid mediator class switching ${ }^{19,20,22}$ and proresolving metabolites. ${ }^{2,21,65}$ Both of these pathways are targets for pharmaceutical drugs for the treatment of inflammation. ${ }^{67}$ Further, it has been proposed that there is a "switch" from the production of proinflammatory prostaglandins to proresolving lipid mediators, leading to programmed resolution of inflammation. ${ }^{4,19}$ If this switch does not take place, a state of chronic inflammation persists.

Depending on the cell type, several prostaglandin synthases and lipoxygenases were upregulated following PEMF treatment. These results are counter to the mechanism of action of pharmaceutical therapeutics such as nonsteroidal anti-inflammatory drugs. We hypothesize the anti-inflammatory effects of PEMF therapy may act primarily 
through a lipid mediator mechanism, such as that proposed by Serhan et al, ${ }^{2,10,21}$ namely through induction of proresolving lipid mediators (eg, resolvins) via a prostaglandin synthase/ lipoxygenase-mediated pathway. A summary of data from the current study in the context of such a model is provided in Figure 7.

In addition to the proresolving pathways, there are distinct anti-inflammatory genetic programs involved in returning tissue to physiological homeostasis. ${ }^{19,68}$ Anti-inflammatory pathways involve mediators, such as interleukin 10 and transforming growth factor beta 1 , which may in fact be induced by proresolving pathways. These cytokines have a dampening effect on the inflammatory response and in some instances, decrease proinflammatory cytokines, such as interleukin 1 beta and tumor necrosis factor alpha.$^{69}$ We have shown, here and previously, that PEMF treatment is followed by a decrease in the expression of interleukin 1 beta and tumor necrosis factor alpha, ${ }^{48}$ as well as an increase in levels of interleukin 10 mRNA, further suggesting an anti-inflammatory mechanism of action for this therapy.
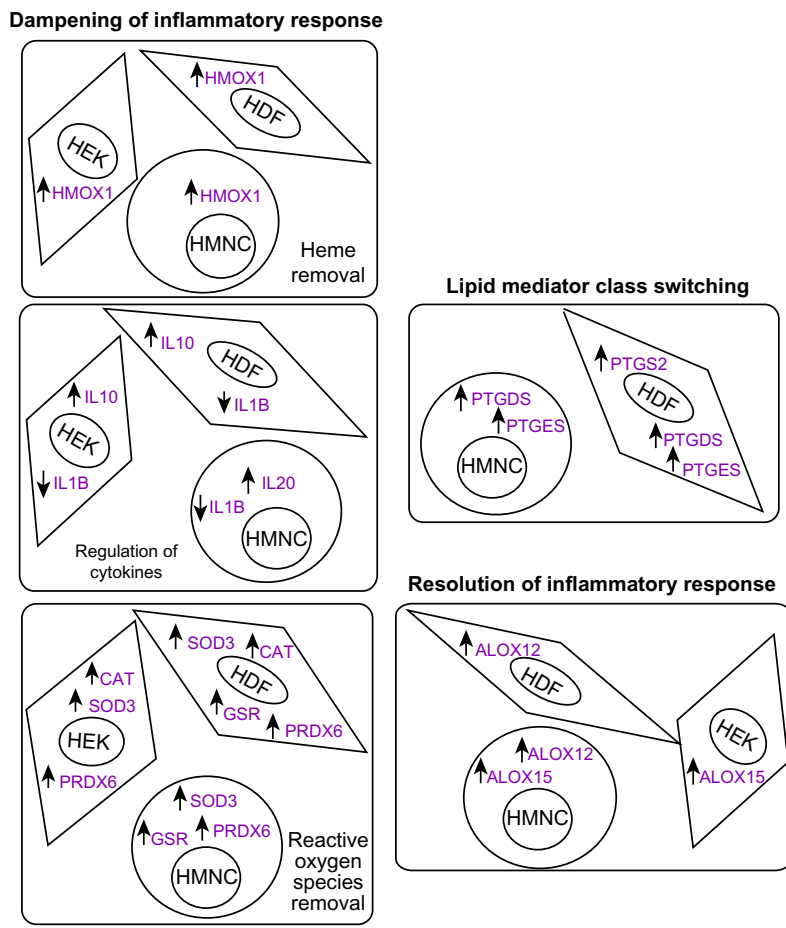

Figure 7 Summary of experimental results from the current study in the context of a possible model.

Notes: In the current study, PEMF treatment was followed by changes in relative amounts of mRNAs encoding factors associated with anti-inflammatory and proresolution effects. An increase or decrease in mRNA levels detected following PEMF treatment, relative to untreated control cells, is indicated by upward- and downward-facing arrows, respectively.

Abbreviations: HDF, human dermal fibroblasts; HEK, human epidermal keratinocytes; HMNC, human mononuclear cells; PEMF, pulsed electromagnetic field; mRNA, messenger RNA.

\section{Conclusion}

Understanding the cellular and molecular mechanisms of inflammation is important for the development of new therapies to treat important health conditions such as inflammatory pain and chronic inflammation. PEMF is a biophysical therapeutic modality based on the delivery of pulsed electromagnetic fields. Although it has been in use for over several decades for the treatment of inflammation, little is known about its biological mechanism of action. Findings from the current study support previously reported data, suggesting PEMF treatment leads to gene expression changes of the factors involved in regulating inflammation, including inflammation resolution. Based on these results, we hypothesize that PEMF-associated analgesia reported in clinical studies may be due to such gene expression changes. Further studies are warranted to further test this hypothesis and to gain additional insight into the mechanisms of action of this novel analgesic modality.

\section{Disclosure}

JM and LMF are employees of Regenesis Biomedical, Inc., Scottsdale, AZ, USA. NJK is a paid consultant for Regenesis Biomedical, Inc. This project was funded by Regenesis Biomedical, Inc. The authors report no other conflicts of interest in this work.

\section{References}

1. Medzhitov R. Inflammation 2010: new adventures of an old flame. Cell. 2010;140(6):771-776.

2. Serhan CN, Chiang N, Van Dyke TE. Resolving inflammation: dual antiinflammatory and pro-resolution lipid mediators. Nat Rev Immunol. 2008;8(5):349-361.

3. Serhan CN, Yacoubian S, Yang R. Anti-inflammatory and proresolving lipid mediators. Annu Rev Pathol. 2008;3:279-312.

4. Buckley CD, Gilroy DW, Serhan CN, Stockinger B, Tak PP. The resolution of inflammation. Nat Rev Immunol. 2013;13(1):59-66.

5. Basbaum AI, Bautista DM, Scherrer G, Julius D. Cellular and molecular mechanisms of pain. Cell. 2009;139(2):267-284.

6. Greene ER, Huang S, Serhan CN, Panigrahy D. Regulation of inflammation in cancer by eicosanoids. Prostaglandins Other Lipid Mediat. 2011;96(1-4):27-36.

7. Vilcek J. The cytokines: an overview. In: Thomson AW, Lotze MT, editors. The Cytokine Handbook. San Diego, CA: Academic Press; 2003:3-18.

8. Yang R, Chiang N, Oh SF, Serhan CN. Metabolomics-lipidomics of eicosanoids and docosanoids generated by phagocytes. Curr Protoc Immunol. 2011; Chapter 14:Unit 14.26.

9. Yang Y, Bazhin AV, Werner J, Karakhanova S. Reactive oxygen species in the immune system. Int Rev Immunol. 2013;32(3):249-270.

10. Serhan CN. Novel lipid mediators and resolution mechanisms in acute inflammation: to resolve or not? Am J Pathol. 2010;177(4):1576-1591.

11. McIntyre TM, Modur V, Prescott SM, Zimmerman GA. Molecular mechanisms of early inflammation. Thromb Haemost. 1997;78(1): 302-305.

12. Mosser DM, Zhang X. Interleukin-10: new perspectives on an old cytokine. Immunol Rev. 2008;226:205-218. 
13. Dutra FF, Bozza MT. Heme on innate immunity and inflammation. Front Pharmacol. 2014;5:115.

14. Wagener FA, van Beurden HE, von den Hoff JW, Adema GJ, Figdor CG. The heme-heme oxygenase system: a molecular switch in wound healing. Blood. 2003;102(2):521-528.

15. Wagener FA, Volk HD, Willis D, et al. Different faces of the hemeheme oxygenase system in inflammation. Pharmacol Rev. 2003;55(3): 551-571.

16. Haworth $\mathrm{O}$, Buckley $\mathrm{CD}$. Resolving the problem of persistence in the switch from acute to chronic inflammation. Proc Natl Acad Sci U SA. 2007;104(52):20647-20648.

17. Kunkel SL, Ogawa H, Conran PB, Ward PA, Zurier RB. Suppression of acute and chronic inflammation by orally administered prostaglandins. Arthritis Rheum. 1981;24(9):1151-1158.

18. Pons F, Williams TJ, Kirk SA, McDonald F, Rossi AG. Pro-inflammatory and anti-inflammatory effects of the stable prostaglandin D2 analogue, ZK 118.182. Eur J Pharmacol. 1994;261(3):237-247.

19. Serhan CN. Pro-resolving lipid mediators are leads for resolution physiology. Nature. 2014;510(7503):92-101.

20. Levy BD, Clish CB, Schmidt B, Gronert K, Serhan CN. Lipid mediator class switching during acute inflammation: signals in resolution. Nat Immunol. 2001;2(7):612-619.

21. Serhan CN, Dalli J, Colas RA, Winkler JW, Chiang N. Protectins and maresins: New pro-resolving families of mediators in acute inflammation and resolution bioactive metabolome. Biochim Biophys Acta. Epub August 17, 2014.

22. Mancini AD, Di Battista JA. The cardinal role of the phospholipase A(2)/ cyclooxygenase-2/prostaglandin E synthase/prostaglandin E(2) (PCPP) axis in inflammostasis. Inflamm Res. 2011;60(12):1083-1092.

23. Serhan CN, Yang R, Martinod K, et al. Maresins: novel macrophage mediators with potent antiinflammatory and proresolving actions. $J$ Exp Med. 2009;206(1):15-23.

24. Spite M, Clària J, Serhan CN. Resolvins, specialized proresolving lipid mediators, and their potential roles in metabolic diseases. Cell Metab. 2014;19(1):21-36

25. Xu ZZ, Zhang L, Liu T, et al. Resolvins RvE1 and RvD1 attenuate inflammatory pain via central and peripheral actions. Nat Med. 2010;16(5):592-597.

26. Ji RR, Xu ZZ, Strichartz G, Serhan CN. Emerging roles of resolvins in the resolution of inflammation and pain. Trends Neurosci. 2011;34(11):599-609.

27. Medzhitov R. Origin and physiological roles of inflammation. Nature. 2008;454(7203):428-435.

28. Brennan FM, Maini RN, Feldmann M. Cytokine expression in chronic inflammatory disease. Br Med Bull. 1995;51(2):368-384.

29. Nathan C, Ding A. Nonresolving inflammation. Cell. 2010;140(6): 871-882.

30. Yeretssian G, Labbé K, Saleh M. Molecular regulation of inflammation and cell death. Cytokine. 2008;43(3):380-390.

31. Stein C, Clark JD, Oh U, et al. Peripheral mechanisms of pain and analgesia. Brain Res Rev. 2009;60(1):90-113.

32. Apfelbaum JL, Chen C, Mehta SS, Gan TJ. Postoperative pain experience: results from a national survey suggest postoperative pain continues to be undermanaged. Anesth Analg. 2003;97(2):534-540.

33. Süleyman H, Demircan B, KaragözY. Anti-inflammatory and side effects of cyclooxygenase inhibitors. Pharmacol Rep. 2007;59(3):247-258.

34. Hasegawa A, Takasaki W, Greene MI, Murali R. Modifying TNFalpha for therapeutic use: a perspective on the TNF receptor system. Mini Rev Med Chem. 2001;1(1):5-16.

35. Moreland LW. Inhibitors of tumor necrosis factor: new treatment options for rheumatoid arthritis. Cleve Clin J Med. 1999;66(6):367-374.

36. Moreland LW. Inhibitors of tumor necrosis factor for rheumatoid arthritis. J Rheumatol Suppl. 1999;57:7-15.

37. Akan Z, Aksu B, Tulunay A, Bilsel S, Inhan-Garip A. Extremely low-frequency electromagnetic fields affect the immune response of monocyte-derived macrophages to pathogens. Bioelectromagnetics. 2010;31(8):603-612.
38. Harper WL, Schmidt WK, Kubat NJ, Isenberg RA. An open-label pilot study of pulsed electromagnetic field therapy in the treatment of failed back surgery syndrome pain. Int Med Case Rep J. 2015;8: $13-22$.

39. Hedén P, Pilla AA. Effects of pulsed electromagnetic fields on postoperative pain: a double-blind randomized pilot study in breast augmentation patients. Aesthetic Plast Surg. 2008;32(4):660-666.

40. Rawe IM, Lowenstein A, Barcelo CR, Genecov DG. Control of postoperative pain with a wearable continuously operating pulsed radiofrequency energy device: a preliminary study. Aesthetic Plast Surg. 2012;36(2):458-463.

41. Rohde C, Chiang A, Adipoju O, Casper D, Pilla AA. Effects of pulsed electromagnetic fields on interleukin-1 beta and postoperative pain: a double-blind, placebo-controlled, pilot study in breast reduction patients. Plast Reconstr Surg. 2010;125(6):1620-1629.

42. Selvam R, Ganesan K, Narayana Raju KV, Gangadharan AC, Manohar BM, Puvanakrishnan R. Low frequency and low intensity pulsed electromagnetic field exerts its antiinflammatory effect through restoration of plasma membrane calcium ATPase activity. Life Sci. 2007;80(26): 2403-2410.

43. Markov M, Nindl G, Hazelwood C, Cuppen J. Interactions between electromagnetic fields and immune system: possible mechanism for pain control. In: Ayrapetyan SN, Markov MS, editors. Bioelectromagnetics Current Concepts. Dordrecht: Springer; 2006:213-225.

44. Guo L, Kubat NJ, Nelson TR, Isenberg RA. Meta-analysis of clinical efficacy of pulsed radio frequency energy treatment. Ann Surg. 2012;255(3):457-467.

45. Guo L, Kubat NJ, Isenberg RA. Pulsed radio frequency energy (PRFE) use in human medical applications. Electromagn Biol Med.2011;30(1): $21-45$.

46. Ball RD. The science of conventional and water-cooled monopolar lumbar radiofrequency rhizotomy: an electrical engineering point of view. Pain Physician. 2014;17(2):E175-E211.

47. Moffett J, Kubat NJ, Griffin NE, Ritz MC, George FR. Pulsed radio frequency energy field treatment of cells in culture: Increased expression of genes involved in angiogenesis and tissue remodeling during wound healing. The Journal of Diabetic Foot Complications. 2011;3(2): 30-39.

48. Moffett J, Fray LM, Kubat NJ. Activation of endogenous opioid gene expression in human keratinocytes and fibroblasts by pulsed radiofrequency energy fields. J Pain Res. 2012;5:347-357.

49. Pilla AA, Muehsam DJ, Markov MS, Sisken BF. EMF signals and ion/ ligand binding kinetics: prediction of bioeffective waveform parameters. Bioelectrochem Bioenerg. 1999;48(1):27-34.

50. Ross CL, Harrison BS. Effect of pulsed electromagnetic field on inflammatory pathway markers in RAW 264.7 murine macrophages. J Inflamm Res. 2013;6:45-51.

51. Ross CL, Harrison BS. Effect of time-varied magnetic field on inflammatory response in macrophage cell line RAW 264.7. Electromagn Biol Med. 2013;32(1):59-69.

52. He YL, Liu DD, Fang YJ, Zhan XQ, Yao JJ, Mei YA. Exposure to extremely low-frequency electromagnetic fields modulates $\mathrm{Na}+$ currents in rat cerebellar granule cells through increase of AA/PGE2 and EP receptor-mediated cAMP/PKA pathway. PLoS One. 2013;8(1): e54376.

53. PillaAA. Nonthermal electromagnetic fields: from first messenger to therapeutic applications. Electromagn Biol Med. 2013;32(2):123-136.

54. Livak KJ, Schmittgen TD. Analysis of relative gene expression data using real-time quantitative PCR and the 2(-Delta Delta C(T)) Method. Methods. 2001;25(4):402-408.

55. Novo E, Parola M. Redox mechanisms in hepatic chronic wound healing and fibrogenesis. Fibrogenesis Tissue Repair. 2008;1(1):5.

56. Cho YS, Song JS, Huh JY, Kim CH, Gong YD, Cheon HG. Discovery of (2-fluoro-benzyl)-(2-methyl-2-phenethyl-2H-chromen-6-yl)-amine (KRH-102140) as an orally active 5-lipoxygenase inhibitor with activity in murine inflammation models. Pharmacology. 2011;87(1-2): $49-55$. 
57. Moffett J, Griffen NE, Ritz MC, George FR. Pulsed radio frequency energy field treatment of cells in culture results in increased expression of genes involved in the inflammation phase of lower extremity diabetic wound healing. The Journal of Diabetic Foot Complications. 2010;2(3):57-64.

58. Medzhitov R, Horng T. Transcriptional control of the inflammatory response. Nat Rev Immunol. 2009;9(10):692-703.

59. Neher MD, Weckbach S, Flierl MA, Huber-Lang MS, Stahel PF. Molecular mechanisms of inflammation and tissue injury after major trauma - is complement the "bad guy"? J Biomed Sci. 2011;18:90.

60. Baranano DE, Rao M, Ferris CD, Snyder SH. Biliverdin reductase: a major physiologic cytoprotectant. Proc Natl Acad Sci US A. 2002;99(25):16093-16098.

61. Wegiel B, Otterbein LE. Go green: the anti-inflammatory effects of biliverdin reductase. Front Pharmacol. 2012;3:47.

62. Pelletier M, Lepow TS, Billingham LK, Murphy MP, Siegel RM. New tricks from an old dog: mitochondrial redox signaling in cellular inflammation. Semin Immunol. 2012;24(6):384-392.

63. Catalá A. Five decades with polyunsaturated Fatty acids: chemical synthesis, enzymatic formation, lipid peroxidation and its biological effects. J Lipids. 2013;2013:710290.

64. Clark AK, Old EA, Malcangio M. Neuropathic pain and cytokines: current perspectives. J Pain Res. 2013;6:803-814.

65. Buckley CD, Gilroy DW, Serhan CN. Proresolving lipid mediators and mechanisms in the resolution of acute inflammation. Immunity. 2014;40(3):315-327.

66. Serhan CN. Resolution phase of inflammation: novel endogenous antiinflammatory and proresolving lipid mediators and pathways. Annu Rev Immunol. 2007;25:101-137.

67. Leslie M. Inflammation's stop signals. Science. 2015;347(6217): $18-21$.

68. Uddin M, Levy BD. Resolvins: natural agonists for resolution of pulmonary inflammation. Prog Lipid Res. 2011;50(1):75-88

69. Commins S, Steinke JW, Borish L. The extended IL-10 superfamily: IL-10, IL-19, IL-20, IL-22, IL-24, IL-26, IL-28, and IL-29. J Allergy Clin Immunol. 2008;121(5):1108-1111.

70. Gozzelino R, Jeney V, Soares MP. Mechanisms of cell protection by heme oxygenase-1. Annu Rev Pharmacol Toxicol. 2010;50:323-354.

71. Wagener FA, Eggert A, Boerman OC, et al. Heme is a potent inducer of inflammation in mice and is counteracted by heme oxygenase. Blood. 2001;98(6):1802-1811.

72. Araujo JA, Zhang M, Yin F. Heme oxygenase-1, oxidation, inflammation, and atherosclerosis. Front Pharmacol. 2012;3:119.

73. Wagener FA, da Silva JL, Farley T, de Witte T, Kappas A, Abraham NG. Differential effects of heme oxygenase isoforms on heme mediation of endothelial intracellular adhesion molecule 1 expression. J Pharmacol Exp Ther. 1999;291(1):416-423.
74. Foti MC, Amorati R. Non-phenolic radical-trapping antioxidants. J Pharm Pharmacol. 2009;61(11):1435-1448.

75. Otterbein LE, Bach FH, Alam J, et al. Carbon monoxide has antiinflammatory effects involving the mitogen-activated protein kinase pathway. Nat Med. 2000;6(4):422-428.

76. auf dem Keller U, Kümin A, Braun S, Werner S. Reactive oxygen species and their detoxification in healing skin wounds. J Investig Dermatol Symp Proc. 2006;11(1):106-111.

77. Mittal M, Siddiqui MR, Tran K, Reddy SP, Malik AB. Reactive oxygen species in inflammation and tissue injury. Antioxid Redox Signal. 2014;20(7):1126-1167.

78. Deng J, Wang X, Qian F, et al. Protective role of reactive oxygen species in endotoxin-induced lung inflammation through modulation of IL-10 expression. J Immunol. 2012;188(11):5734-5740.

79. Rochette L, Zeller M, Cottin Y, Vergely C. Diabetes, oxidative stress and therapeutic strategies. Biochim Biophys Acta. 2014;1840(9): 2709-2729.

80. Jiang J, Dingledine R. Prostaglandin receptor EP2 in the crosshairs of anti-inflammation, anti-cancer, and neuroprotection. Trends Pharmacol Sci. 2013;34(7):413-423.

81. Nakamura H, Murayama T. Role of sphingolipids in arachidonic acid metabolism. J Pharmacol Sci. 2014;124(3):307-312.

82. Feng L, Xia Y, Garcia GE, Hwang D, Wilson CB. Involvement of reactive oxygen intermediates in cyclooxygenase-2 expression induced by interleukin-1, tumor necrosis factor-alpha, and lipopolysaccharide. J Clin Invest. 1995;95(4):1669-1675.

83. Korbecki J, Baranowska-Bosiacka I, Gutowska I, Chlubek D. The effect of reactive oxygen species on the synthesis of prostanoids from arachidonic acid. J Physiol Pharmacol. 2013;64(4):409-421.

84. Suzuki T, Hashimoto S, Toyoda N, et al. Comprehensive gene expression profile of LPS-stimulated human monocytes by SAGE. Blood. 2000;96(7):2584-2591.

85. Calder PC. Marine omega-3 fatty acids and inflammatory processes: Effects, mechanisms and clinical relevance. Biochim Biophys Acta. Epub August 20, 2014.

86. Rådmark O, Werz O, Steinhilber D, Samuelsson B. 5-Lipoxygenase, a key enzyme for leukotriene biosynthesis in health and disease. Biochim Biophys Acta. Epub August 22, 2014.

87. Simmons DL, Botting RM, Hla T. Cyclooxygenase isozymes: the biology of prostaglandin synthesis and inhibition. Pharmacol Rev. 2004;56(3):387-437.
Journal of Inflammation Research

\section{Publish your work in this journal}

The Journal of Inflammation Research is an international, peer-reviewed open-access journal that welcomes laboratory and clinical findings on the molecular basis, cell biology and pharmacology of inflammation including original research, reviews, symposium reports, hypothesis formation and commentaries on: acute/chronic inflammation; mediators of inflamma-

\section{Dovepress}

tion; cellular processes; molecular mechanisms; pharmacology and novel anti-inflammatory drugs; clinical conditions involving inflammation. The manuscript management system is completely online and includes a very quick and fair peer-review system. Visit http://www.dovepress.com/ testimonials.php to read real quotes from published authors. 